\title{
Near-field thermal radiation between homogeneous dual uniaxial electromagnetic metamaterials
}

Jui-Yung Chang, Soumyadipta Basu, Yue Yang, and Liping Wang

Citation: Journal of Applied Physics 119, 213108 (2016); doi: 10.1063/1.4953253

View online: $\mathrm{http}: / / d x . d o i . o r g / 10.1063 / 1.4953253$

View Table of Contents: http://aip.scitation.org/toc/jap/119/21

Published by the American Institute of Physics

\section{Articles you may be interested in}

Near-field radiative thermal transport: From theory to experiment

AIP Advances 5, 053503 (2015); 10.1063/1.4919048

Near-field radiative heat transfer between metamaterials coated with silicon carbide thin films

Applied Physics Letters 106, 033106 (2015); 10.1063/1.4906530

Near-field radiative heat transfer between doped-Si parallel plates separated by a spacing down to $200 \mathrm{~nm}$ Applied Physics Letters 109, 203112 (2016); 10.1063/1.4967384

Tunable quasi-monochromatic near-field radiative heat transfer in $s$ and $p$ polarizations by a hyperbolic metamaterial layer

Journal of Applied Physics 121, 013106 (2017); 10.1063/1.4973530

Radiation-based near-field thermal rectification with phase transition materials

Applied Physics Letters 103, 163101 (2013); 10.1063/1.4825168

Near-field radiative transfer based thermal rectification using doped silicon

Applied Physics Letters 98, 113106 (2011); 10.1063/1.3567026

\section{AlP | Journal of}

Save your money for your research.

It's now FREE to publish with us no page, color or publication charges apply.

\section{Publish your research in the} Joumal of Applied Physics to claim your place in applied physics history. 


\title{
Near-field thermal radiation between homogeneous dual uniaxial electromagnetic metamaterials
}

\author{
Jui-Yung Chang, Soumyadipta Basu, Yue Yang, and Liping Wang ${ }^{\text {a) }}$ \\ School for Engineering of Matter, Transport and Energy, Arizona State University, Tempe, Arizona 85287, USA
}

(Received 27 March 2016; accepted 23 May 2016; published online 7 June 2016)

\begin{abstract}
Recently, near-field thermal radiation has attracted much attention in several fields since it can exceed the Planck blackbody limit through the coupling of evanescent waves. In this work, near-field radiative heat transfer between two semi-infinite dual uniaxial electromagnetic metamaterials with two different material property sets is theoretically analyzed. The near-field radiative heat transfer is calculated using fluctuational electrodynamics incorporated with anisotropic wave optics. The underlying mechanisms, namely, magnetic hyperbolic mode, magnetic surface polariton, electrical hyperbolic mode, and electrical surface polariton, between two homogeneous dual uniaxial electromagnetic metamaterials are investigated by examining the transmission coefficient and the spectral heat flux. The effect of vacuum gap distance is also studied, which shows that the enhancement at smaller vacuum gap is mainly due to hyperbolic mode and surface plasmon polariton modes. In addition, the results show that the contribution of s-polarized waves is significant and should not be excluded due to the strong magnetic response regardless of vacuum gap distances. The fundamental understanding and insights obtained here will facilitate the finding and application of novel materials for near-field thermal radiation. Published by AIP Publishing. [http://dx.doi.org/10.1063/1.4953253]
\end{abstract}

\section{INTRODUCTION}

Due to the coupling of evanescent waves, near-field thermal radiation can overcome the Planck blackbody limit when the vacuum gap distance between the emitter and receiver is less than the characteristic thermal wavelength of radiation heat transfer. It has become a popular topic in the field of energy harvesting ${ }^{1,2}$ and thermal management. ${ }^{3,4}$ In particular, near-field thermal radiation can be further enhanced by coupling the surface plasmon/phonon polaritons $(\mathrm{SPP} / \mathrm{SPhP})$ of the interfaces on either side of nanometer vacuum gaps. ${ }^{5-7}$ Besides hyperbolic metamaterial (HMM) ${ }^{8,9}$ is another promising mechanism to enhance the near-field radiation known by its unlimited length of wavevector (high$\mathrm{k}$ mode $)^{10,11}$ and broadband enhancement on photonic density of state (PDOS). ${ }^{12,13}$ Even though these two mechanisms have been intensively studied for near-field radiative heat transfer enhancement, most of reported research investigated both SPP/SPhP excitation and HM modes due to the materials electrical response only associated with electrical permittivity, while the magnetic response is usually neglected, or non-magnetic materials are simply assumed. ${ }^{6,7}$

Over past few years, artificial magnetic response or magnetic polariton from nanostructure based metamaterials has been widely investigated for energy applications in the far field. ${ }^{14-17}$ This reveals the possibility of extra modes based on magnetic responses, which can provide additional enhancement on near-field thermal radiation. Recent studies have shown that magnetic surface modes can also be excited for transverse electric (TE) waves based on the effective negative permeability of metamaterials, which leads to

\footnotetext{
a) Author to whom correspondence should be addressed. Electronic mail: liping.wang@asu.edu. Tel.: 1-480-727-8615.
}

additional near-field transfer enhancement in both wave polarizations, but only isotropic materials were considered. ${ }^{18}$

The present study focuses on the theoretical analysis of near-field radiative heat transfer between two semi-infinite dual uniaxial electromagnetic metamaterials. In order to reveal the possible additional modes for enhancing near-field thermal radiation, two homogeneous media with two different material property sets will be investigated. The near-field radiative heat transfer is calculated by fluctuational electrodynamics incorporated with anisotropic wave optics by taking into account the electromagnetic responses to both $\mathrm{s}$ and p polarized waves. Enhancement modes along with the associated mechanisms will be understood by the energy transmission coefficients and spectral heat fluxes for different material property sets. Furthermore, the effect of vacuum gap distance on the total heat flux will be discussed at different wave polarizations.

\section{THEORETICAL METHODS}

\section{A. Material property sets}

Figure 1 depicts near-field radiative heat transfer between two semi-infinite homogeneous metamaterials separated by a vacuum gap $d$ to be analyzed in this study. The emitter (Layer 1) is kept at $400 \mathrm{~K}$ and the receiver (Layer 2) is at $300 \mathrm{~K}$. The electromagnetic metamaterials are considered to be identical for simplicity, while both respond to electrical and magnetic fields with permittivity $\varepsilon$ and permeability $\mu$, respectively, expressed by Drude and Lorentz models: ${ }^{18,19}$ The electromagnetic metamaterials are considered identical for simplicity, while both respond to electrical and magnetic fields with permittivity $\varepsilon$ and permeability $\mu$ that are expressed by the Drude and Lorentz models ${ }^{18,19}$ 


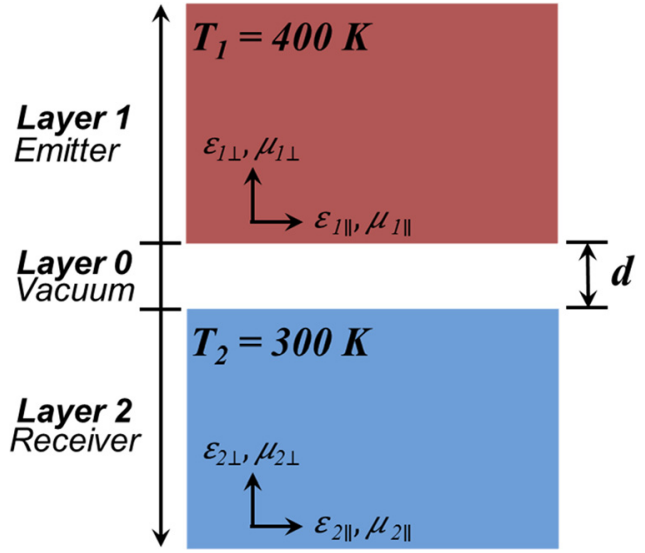

FIG. 1. Schematic of two homogeneous semi-infinite uniaxial electromagnetic metamaterials at different temperatures separated by a nanometer vacuum gap $d$.

$$
\varepsilon=1-\frac{\omega_{p}^{2}}{\omega^{2}+i \omega \gamma_{e}},
$$

and

$$
\mu=1-\frac{F \omega^{2}}{\omega^{2}-\omega_{0}^{2}+i \omega \gamma_{m}},
$$

where $F=0.56$ is the split ring filling ratio factor, $\omega_{\mathrm{p}}=10^{14} \mathrm{rad} / \mathrm{s}$ is the equivalent plasma frequency, $\omega_{0}=0.4 \omega_{\mathrm{p}}$ is the effective resonance frequency, and $\gamma_{\mathrm{e}}=\gamma_{\mathrm{m}}=0.01 \omega_{\mathrm{p}}$ are the electrical and magnetic scattering rates, respectively. ${ }^{18}$ Figure 2 plots the real parts of the electrical permittivity $\varepsilon$ and magnetic permeability $\mu$, respectively, expressed by Drude and Lorentz models. Clearly, the electromagnetic metamaterial exhibits metallic behavior, i.e., $\operatorname{Re}(\varepsilon)<0$, only at lower frequencies $\omega<\omega_{\mathrm{p}}$. On the other hand, it shows negative magnetic response with $\operatorname{Re}(\mu)<0$ within a narrow frequency band of $\omega_{0}<\omega<$

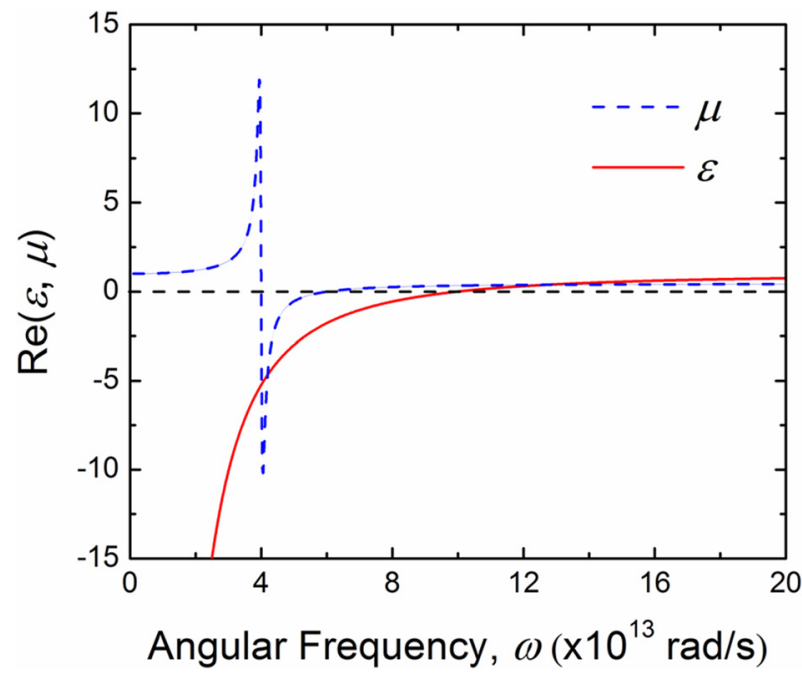

FIG. 2. The real parts of electrical permeability $\varepsilon$ and magnetic permittivity $\mu$ respectively obtained by the Drude and Lorentz models described by Eqs. (1) and (2). Used parameters are: split ring filling ratio factor $F=0.56$, equivalent plasma frequency $\omega_{\mathrm{p}}=10^{14} \mathrm{rad} / \mathrm{s}$, effective resonance frequency $\omega_{0}=0.4 \omega_{\mathrm{p}}$, and electrical and magnetic scattering rates $\gamma_{\mathrm{e}}=\gamma_{\mathrm{m}}=0.01 \omega_{\mathrm{p}}$. $\omega_{0} / \sqrt{1-F}$ when $\gamma_{m} \ll \omega$. Note that when the uniaxial material properties are described by effective medium theory, the vacuum gap should be larger than the unit size of the metamaterial. $^{20}$

In order to study the effect of anisotropic material properties on the near-field radiative transfer, both media are assumed to be uniaxial with ordinary permittivity and permeability components following the Drude an Lorentz models, i.e., $\varepsilon_{\|}=\varepsilon$ and $\mu_{\|}=\mu$, while the extraordinary permittivity and permeability components being either 1 or -1 , i.e., $\varepsilon_{\perp}=\mu_{\perp}=1$ or -1 . These two different property sets of homogenous uniaxial electromagnetic metamaterials, also listed in Table I, are considered here to study possible nearfield enhancement mechanisms because of the responses to both electrical and magnetic fields.

\section{B. Near-field radiative heat transfer between uniaxial electromagnetic metamaterials}

In this work, we extend the fluctuational electrodynamics theory exclusively for uniaxial metamaterials that respond to both electrical and magnetic fields. The net radiative heat flux between two semi-infinite dual uniaxial media at temperatures of $T_{1}$ and $T_{2}$ is given by ${ }^{21}$

$$
\begin{aligned}
q^{\prime \prime} & =\int_{0}^{\infty} q(\omega) d \omega \\
& =\frac{1}{4 \pi^{2}} \int_{0}^{\infty} d \omega\left[\Theta\left(\omega, T_{1}\right)-\Theta\left(\omega, T_{2}\right)\right] \int_{0}^{\infty} \beta \xi(\omega, \beta) d \beta
\end{aligned}
$$

where $\Theta(\omega, T)$ is the spectral mean energy of a Planck oscillator at absolute temperature $T$ and angular frequency $\omega$,

$$
\Theta(\omega, T)=\frac{\hbar \omega}{\exp \left(\hbar \omega / k_{B} T\right)-1} .
$$

Here, $\hbar, k_{B}$, and $\beta$ are the reduced Planck constant, the Boltzmann constant, and the component of the wavevector parallel to the interface, respectively. The expression for energy transmission coefficient $\xi(\omega, \beta)$ is different for propagating waves $(\beta<\omega / c)$ and evanescent waves $(\beta>\omega / c)$, both of which consist of two contributions from $\mathrm{s}$ and $\mathrm{p}$ polarized waves ${ }^{22}$

$$
\begin{aligned}
\xi_{\text {prop }}(\omega, \beta)= & \frac{\left(1-\left|r_{01}^{s}\right|^{2}\right)\left(1-\left|r_{02}^{s}\right|^{2}\right)}{\left|1-r_{01}^{s} r_{02}^{s} e^{i 2 \gamma_{0} d}\right|^{2}} \\
& +\frac{\left(1-\left|r_{01}^{p}\right|^{2}\right)\left(1-\left|r_{02}^{p}\right|^{2}\right)}{\left|1-r_{01}^{p} r_{02}^{p} e^{i 2 \gamma_{0} d}\right|^{2}},
\end{aligned}
$$

and

TABLE I. Two different property sets of homogeneous uniaxial electromagnetic metamaterials to be studied for near-field radiative heat transfer.

\begin{tabular}{lcccc}
\hline \hline Property sets & $\varepsilon_{\|}, \varepsilon_{2 \|}$ & $\varepsilon_{1 \perp}, \varepsilon_{2 \perp}$ & $\mu_{1 \|}, \mu_{2 \|}$ & $\mu_{1 \perp}, \mu_{2 \perp}$ \\
\hline I & $\varepsilon$ & 1 & $\mu$ & 1 \\
II & $\varepsilon$ & -1 & $\mu$ & -1 \\
\hline \hline
\end{tabular}




$$
\begin{aligned}
\xi_{\text {evan }}(\omega, \beta)= & \frac{4 \operatorname{Im}\left(r_{01}^{s}\right) \operatorname{Im}\left(r_{02}^{s}\right) e^{-2 \operatorname{Im}\left(\gamma_{0}\right) d}}{\left|1-r_{01}^{s} r_{02}^{s} e^{i 2 \gamma_{0} d}\right|^{2}} \\
& +\frac{4 \operatorname{Im}\left(r_{01}^{p}\right) \operatorname{Im}\left(r_{02}^{p}\right) e^{-2 \operatorname{Im}\left(\gamma_{0}\right) d}}{\left|1-r_{01}^{p} r_{02}^{p} e^{i 2 \gamma_{0} d}\right|^{2}}
\end{aligned}
$$

where the index 1,0 , and 2 represent the semi-infinite emitter 1 , the vacuum gap layer, and the semi-infinite receiver 2 , respectively. The symbol "Im" indicates the imaginary part of a complex number. $r_{i j}^{s, p}$ is the Fresnel reflection coefficient at the interface between medium $i$ and $j$ for either $\mathrm{s}$ or $\mathrm{p}$ polarized waves (see Appendix A for detailed derivations)

$$
r_{i j}^{s}=\frac{\mu_{j, \|} \gamma_{i}^{s}-\mu_{i, \|} \gamma_{j}^{s}}{\mu_{j, \|} \gamma_{i}^{s}+\mu_{i, \|} \gamma_{j}^{s}},
$$

and

$$
r_{i j}^{p}=\frac{\varepsilon_{j, \|} \gamma_{i}^{p}-\varepsilon_{i, \|} \gamma_{j}^{p}}{\varepsilon_{j, \|} \gamma_{i}^{p}+\varepsilon_{i, \|} \gamma_{j}^{p}} .
$$

Here, $\gamma_{i}^{s, p}$ is the $\mathrm{z}$-component of the wavevector expressed under different wave polarizations (see Appendix B for detailed derivations)
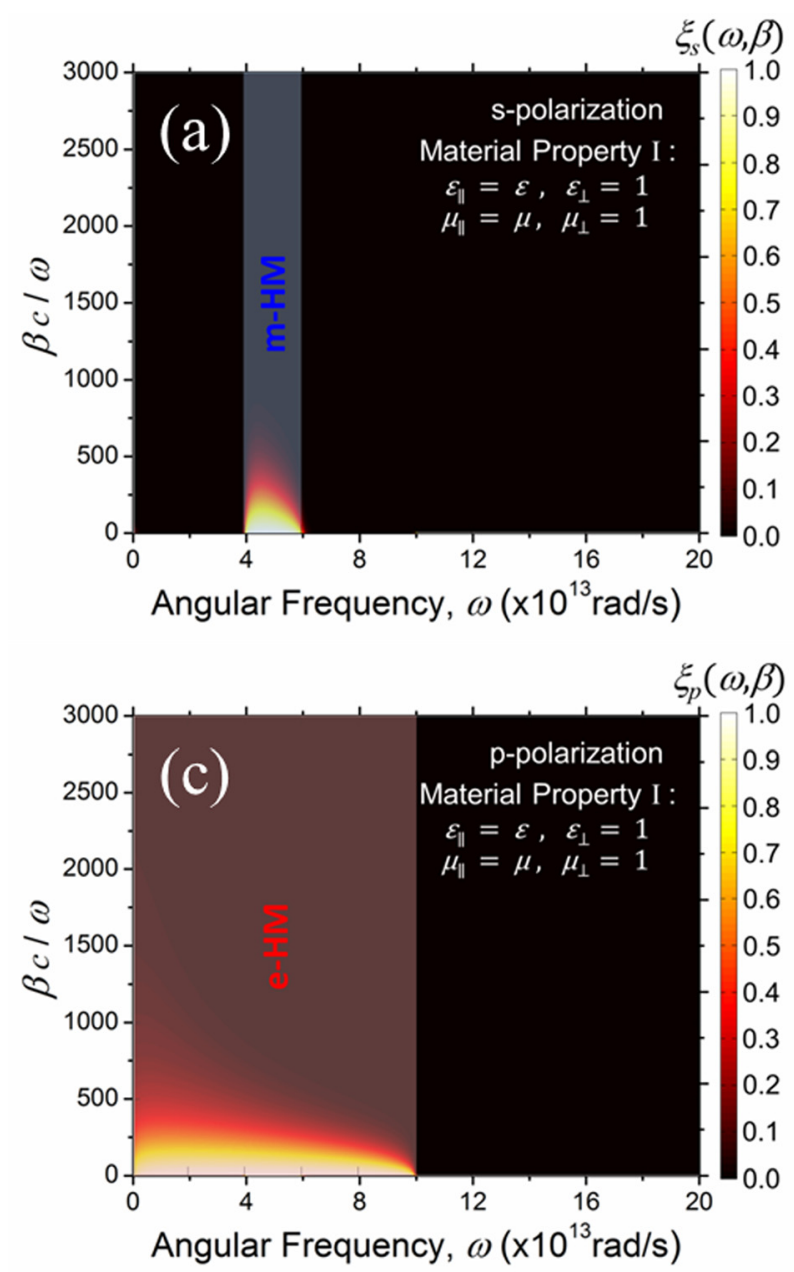

$$
\gamma_{i}^{p}=\sqrt{\varepsilon_{i, \|} \mu_{i, \|} \frac{\omega^{2}}{c^{2}}-\frac{\varepsilon_{i, \|}}{\varepsilon_{i, \perp}} \beta_{i}{ }^{2}},
$$

and

$$
\gamma_{i}^{p}=\sqrt{\varepsilon_{i, \|} \mu_{i, \|} \frac{\omega^{2}}{c^{2}}-\frac{\varepsilon_{i, \|}}{\varepsilon_{i, \perp}} \beta^{2}},
$$

where $c$ is the speed of light in vacuum. Note that, due to the uniaxial wave optics, for s polarized waves, only $\varepsilon_{\|}$determines the electrical responses while both $\mu_{\|}$and $\mu_{\perp}$ are involved with magnetic response. On the other hand, for $\mathrm{p}$ polarized waves, both $\varepsilon_{\|}$and $\varepsilon_{\perp}$ affect the electrical response, while only $\mu_{\|}$solely represents magnetic response.

\section{RESULTS AND DISCUSSION}

\section{A. Transmission coefficients at different wave polarizations}

In order to analyze the possible enhancement mechanisms between uniaxial electromagnetic metamaterials, the transmission coefficients $\xi$ as a function of both angular frequency $(\omega)$ and normalized parallel wavevector component $(\beta c / \omega)$ are first investigated. Figures 3(a) and 3(b) show the
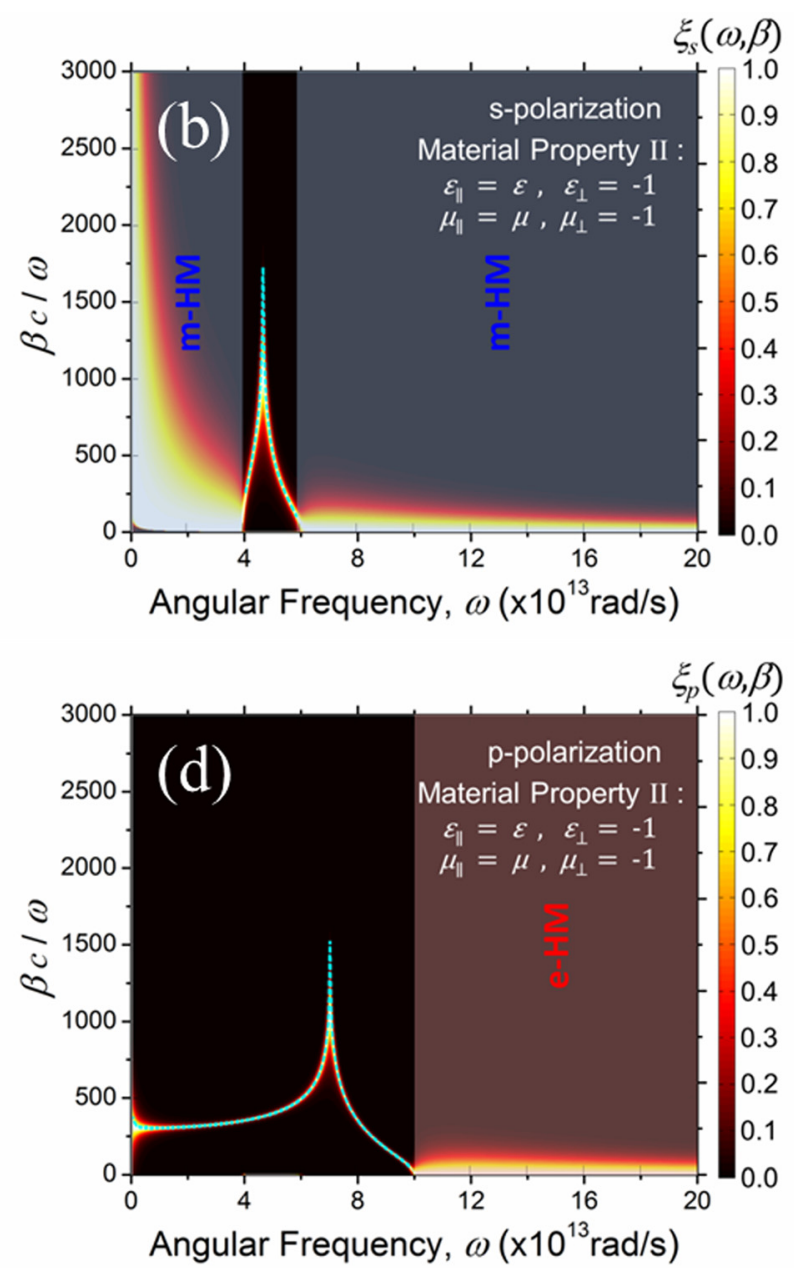

FIG. 3. The transmission coefficient of the near-field radiative heat transfer between two semi-infinite uniaxial electromagnetic metamaterials for s polarized waves with material property sets of: (a) I; (b) II and for p polarized waves with material property sets of: (c) I; (d) II. The vacuum gap distance is $d=20$ nm. 
transmission coefficients $\xi(\omega, \beta)$ of property sets I and II under $\mathrm{s}$ polarized waves. Note that, under s polarization, only $\varepsilon_{\|}$, which follows the Drude behavior, is involved for the electrical response, while both $\mu_{\|}$and $\mu_{\perp}$ have to be considered for the magnetic response.

In Fig. 3(a) for the property set I, near-field energy transmission enhancement is observed under the shaded region within the frequency range between $4 \times 10^{13} \mathrm{rad} / \mathrm{s}<\omega<6$ $\times 10^{13} \mathrm{rad} / \mathrm{s}$, which is corresponding to the $\operatorname{Re}\left(\mu_{\|}=\mu\right)<0$ region (i.e., $\omega_{0}<\omega<\omega_{0} / \sqrt{1-F}$ ). Apparently, as $\mu_{\perp}=1$ or as long as $\mu_{\perp}$ is positive, the near-field enhancement within this narrow spectral band is due to the type 2 (i.e., $\mu_{\|}<0, \mu_{\perp}>0$ ) magnetic hyperbolic mode (m-HM). Outside of the shaded m-HM region, both $\mu_{\perp}$ and $\mu_{\|}$are positive. Therefore, no other enhancement modes are supported, and the energy transmission is weak as observed.

For the property set II with $\mu_{\perp}=-1$ instead of 1 , as shown in Fig. 3(b), two broad enhancement bands are observed at frequencies $\omega<4 \times 10^{13} \mathrm{rad} / \mathrm{s}$ and $\omega>6 \times 10^{13}$ $\mathrm{rad} / \mathrm{s}$, in which $\operatorname{Re}\left(\mu_{\|}\right)$is positive. Therefore, the broadband near-field enhancements at $\omega<\omega_{0}$ and $\omega>\omega_{0} / \sqrt{1-F}$ are due to the type $1 \mathrm{~m}-\mathrm{HM}$ mode as long as $\mu_{\|}>0$ and $\mu_{\perp}<0$. Besides, within the negative magnetic response region $\operatorname{Re}\left(\mu_{\|}\right)<0$, there exist additional enhancement modes that strongly depend on both frequency $\omega$ and wavevector $\beta$. By plotting the analytical dispersion relation of coupled magnetic SPP ( $\mathrm{m}$-SPP) between the uniaxial metamaterials (blue dashed line in the figure), which is obtained through zeroing the denominator of the transmission coefficient for s polarized waves, i.e., $1-r_{01}^{s} r_{02}^{s} e^{i 2 \gamma_{0} d}=0$, the perfect agreement with the enhancement bands from the energy transmission coefficient contour undoubtedly confirms the physical mechanism as coupled $\mathrm{m}$-SPP mode. The low and high frequency coupled $\mathrm{m}$-SPP branches merge at the asymptotic frequency where $\mu_{\|} \mu_{\perp}=1$, which is exactly the resonance frequency of $\mathrm{m}$-SPP at a single interface between the uniaxial metamaterial and vacuum (see Appendix $\mathrm{C}$ for detailed derivations).

Figures 3(c) and 3(d) show the transmission coefficients for both material property sets I and II but under p polarized waves, where only $\varepsilon_{\|}, \varepsilon_{\perp}$, and $\mu_{\|}$are involved for the electrical and magnetic field responses. For the property set I with $\varepsilon_{\perp}=1$, a broad enhancement band exists in the energy transmission coefficient contour at low frequencies $\omega<\omega_{\mathrm{p}}=1$ $\times 10^{14} \mathrm{rad} / \mathrm{s}$, where $\varepsilon_{\|}<0$ and $\varepsilon_{\perp}>0$, indicating the type 2 electrical hyperbolic mode (e-HM). Likewise, there is no near-field enhancement at high frequency range due to the dielectric behavior of the property set I with both positive $\operatorname{Re}\left(\varepsilon_{\|}\right)$and $\operatorname{Re}\left(\varepsilon_{\perp}\right)$. Note that, enhancements due to the frustrated total internal reflection ${ }^{8}$ still exist when the materials express dielectric behavior, but this effect cannot be seen here since it supports wavevector only at $\beta<\sqrt{\varepsilon_{i, \perp}} \mu_{i, \|} \omega / c$ according to Eq. (9).

As shown in Fig. 3(d) for the property set II with $\varepsilon_{\perp}=-1$ under $\mathrm{p}$ polarized waves, there exists broadband enhancement at high frequencies $\omega>\omega_{\mathrm{p}}=1 \times 10^{14} \mathrm{rad} / \mathrm{s}$ due to type $1 \mathrm{e}-\mathrm{HM}$ mode with $\varepsilon_{\|}>0$ and $\varepsilon_{\perp}<0$, as well as coupled electrical SPP (e-SPP) mode at low frequency region $\omega<\omega_{\mathrm{p}}$, in comparison to the narrow m-SPP mode excited by s polarized waves in Fig. 3(b). The dispersion curves of coupled e-SPP modes are also shown by solving $1-r_{01}^{p} r_{02}^{p} e^{i 2 \gamma_{0} d}=0$. Similarly, the two branches of coupled e-SPP modes merges at the asymptotic frequency for that at a single interface where $\varepsilon_{\|} \varepsilon_{\perp}=1$ (see Appendix $\mathrm{C}$ for detailed derivation).

Note that the enhancement bands associated with $\mathrm{m}-\mathrm{HM}$ at s polarization and e-HM modes at p polarization are complementary and cover the entire frequency spectra for both property sets I and II. Furthermore, SPP modes would never occur for the property set I with $\mu_{\perp}=\varepsilon_{\perp}=1$ at either s or $\mathrm{p}$ polarizations as positive $\mu_{\perp}$ and $\varepsilon_{\perp}$ cannot satisfy the m-SPP or e-SPP excitation conditions. On the other hand, for the property set II with $\mu_{\perp}=\varepsilon_{\perp}=-1$, both m-SPP and e-SPP modes can be excited, respectively, under $\mathrm{s}$ and p polarized waves. Note that the conditions provided here are only for coupled SPP modes between the same materials where the asymptotic frequencies are the same as those at the single interface SPP modes given by $\mu_{\|} \mu_{\perp}=1$ for m-SPP and $\varepsilon_{\|} \varepsilon_{\perp}$ $=1$ for e-SPP between dual uniaxial metamaterials and vacuum. Both conditions are derived through the assumption of $\beta \gg \omega / \mathrm{c}$, which turns out to be respectively regardless of $\varepsilon_{\|}$ and $\mu_{\|}$(see Appendix C).

\section{B. Spectral heat fluxes at different wave polarizations}

The spectral heat fluxes $q(\omega)$ can be obtained by integrating the transmission coefficient over $\beta$ according to Eq. (3). Figures 4(a) and 4(b) present the spectral heat fluxes $q(\omega)$ with different property sets under s and p polarizations, respectively. The green shaded areas indicate the regions where $\operatorname{Re}\left(\mu_{\|}=\mu\right)<0$ at s polarization and $\operatorname{Re}\left(\varepsilon_{\|}=\varepsilon\right)<0$ at p polarization. For the property set I, the spectral heat flux is significantly enhanced only within the shaded regions because of the type $2 \mathrm{~m}-\mathrm{HM}$ and e-HM modes respectively at each $\mathrm{s}$ and $\mathrm{p}$ polarization. As no coupled SPP modes exist, the spectral heat flux abruptly decreases at frequencies outside of the negative $\mu_{\|}$and $\varepsilon_{\|}$regions. On the other hand, for the property set II, the spectral heat flux exhibits strong enhancement within the shaded regions due to coupled mSPP and e-SPP modes which agrees with the negative $\mu_{\|}$and $\varepsilon_{\|}$regions. More importantly, the type $1 \mathrm{~m}-\mathrm{HM}$ and e-HM modes elsewhere exhibit at least 4 orders of magnitude enhancement under both $\mathrm{s}$ and $\mathrm{p}$ polarizations in comparison with the property set I. Note that the coupled SPP modes result in narrow-band spectral heat flux peaks within the negative $\mu_{\|}$and $\varepsilon_{\|}$regions, whose magnitude is even about one order greater than that associated with the broadband type 2 hyperbolic modes with the property set I.

Clearly, these two property sets with either positive or negative $\mu_{\perp}$ and $\varepsilon_{\perp}$ could lead to significantly different spectral heat flux distributions, which would be favored for different applications. For example, the broadband flux enhancement associated with type 2 hyperbolic modes provided by the material property set I or with type 1 hyperbolic modes from the set II could be useful for augmentation or suppression of radiative heat transfer within the spectral range of interests. The narrow-band flux enhancement or wavelength-selective control of near-field radiative transfer due to coupled SPP modes are highly desired for enhancing 

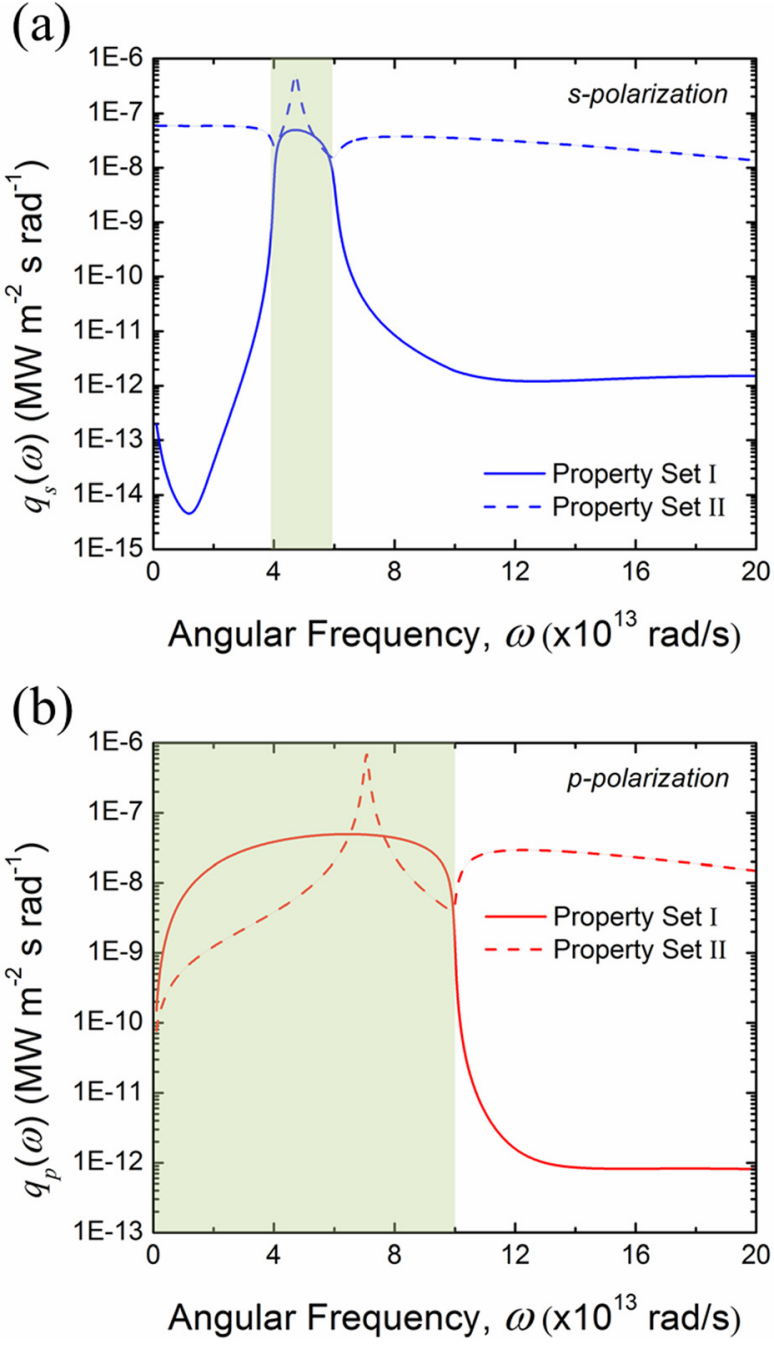

FIG. 4. Spectral heat flux between two semi-infinite uniaxial electromagnetic metamaterials with material property sets of I and II separated by a vacuum gap $d=20 \mathrm{~nm}$ for (a) s polarized waves; (b) p polarized waves.

thermal energy harvesting and conversion applications like thermophotovoltaics. Moreover, the flux enhancement over the entire spectrum by taking advantage of both HM and SPP modes is of great benefit for thermal management and heat dissipation. Besides, by suitably selecting the materials with either Drude or Lorentz behaviors respectively for the electrical permittivity $\varepsilon$ or magnetic permeability $\mu$, these heat flux enhancement or the HM and SPP modes can be activated selectively for either s or $\mathrm{p}$ polarization, which would facilitate polarization-sensitive applications.

\section{Effect of vacuum gap on the total heat flux}

Finally, the effect of vacuum gap distance $(d)$ on the total heat flux is investigated for the two property sets of dual uniaxial metamaterials in comparison with those for two isotropic cases, one of which possesses the electrical permittivity $\varepsilon_{\|}=\varepsilon_{\perp}=\varepsilon$ described by the Drude model in Eq. (1) and magnetic permeability $\mu_{\|}=\mu_{\perp}=1$ (i.e., nonmagnetic) and the other isotropic metamaterial has $\mu_{\|}=\mu_{\perp}=\mu$ given by the Lorentz model in Eq. (2). Figure 5(a) shows the total heat fluxes of four different materials for $\mathrm{s}$ polarized
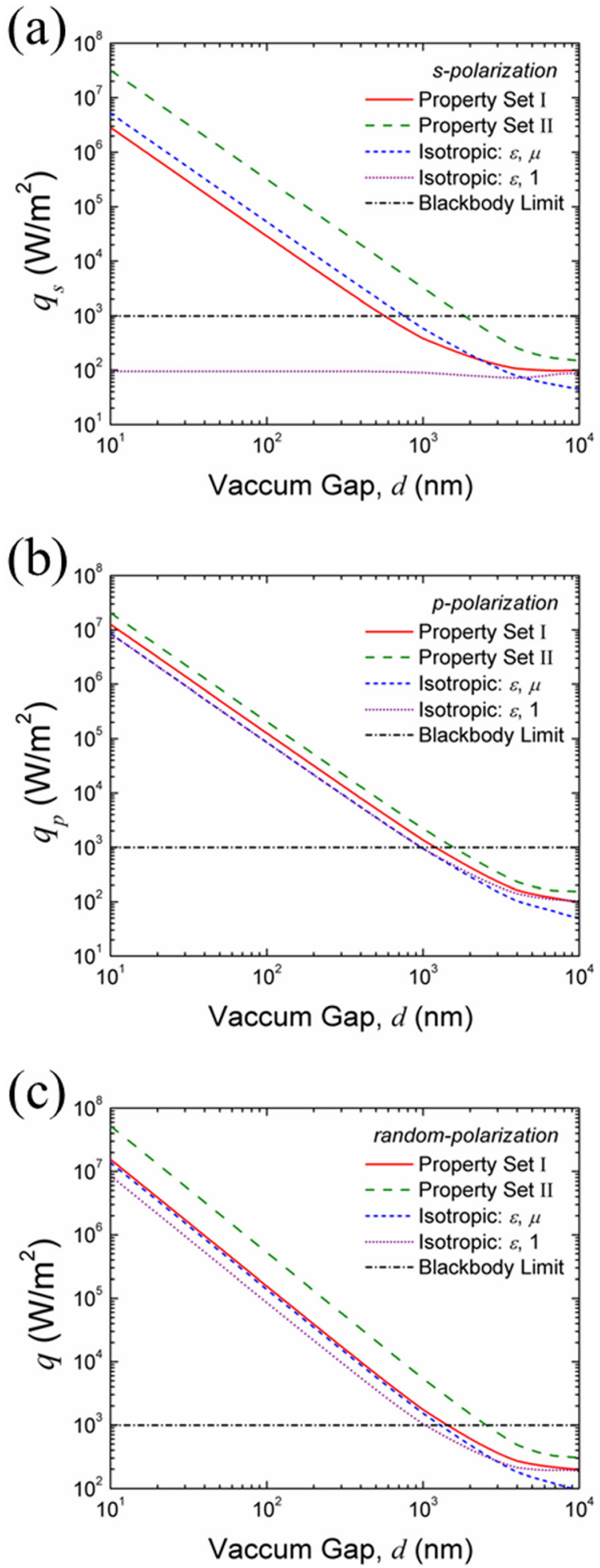

FIG. 5. Normalized total heat fluxes between two semi-infinite uniaxial electromagnetic metamaterials with different sets of material properties with respect to different vacuum gap $d$ for (a) s polarized waves; (b) p polarized waves; (c) randomly polarized waves.

waves as a function of $d$. As the vacuum gap distances decrease from $10 \mu \mathrm{m}$ to $10 \mathrm{~nm}$, the near-field heat fluxes under s polarization for all three metamaterials with magnetic responses increase monotonically and could exceed the far-field blackbody limit by more than 3 orders except for non-magnetic isotropic material $(\varepsilon, \mu=1)$. It is well known 
that the near-field heat flux at s polarized waves between non-magnetic isotropic metals is small due to weak coupling of evanescent waves ${ }^{4,21}$ and in this case, the flux is about one order of magnitude smaller than the blackbody limit and little changes with gap distances. For the isotropic metamaterial $(\varepsilon, \mu)$ with magnetic responses, the s-polarized total heat flux $q$ is much enhanced with $d^{-2}$ dependence due to the excitation of coupled m-SPP modes whose strengths increases significantly at smaller vacuum gaps, as studied in Ref. 18 . However, for the dual uniaxial metamaterials with the property set I, the total heat flux is slightly lower than that for isotropic metamaterials, mainly because only $\mathrm{m}-\mathrm{HM}$ within the limited $\operatorname{Re}\left(\mu_{\|}=\mu\right)<0$ region but no m-SPP modes exist, as shown in Figs. 3(a) and 4(a). Moreover, as the dual uniaxial metamaterials with the property set II could excite both mSPP and type $2 \mathrm{~m}$-HM modes within a broad spectral region, the total heat flux under s polarization can be enhanced to be more than 4 orders greater than the blackbody limit, which is about 1 order more than that for isotropic metamaterials.

Figure 5(b) presents the gap distance effect on the total heat flux for $\mathrm{p}$ polarized waves; while the fluxes for all four materials exhibit $d^{-2}$ dependence as the vacuum gap becomes smaller, exceeding the blackbody limit up to 4 orders of magnitudes. The two isotropic materials $(\varepsilon, \mu=1)$ and $(\varepsilon, \mu)$ have almost the same heat fluxes since for $\mathrm{p}$ polarization, the identical electrical permittivity $\varepsilon$ results in the same coupled-SPP resonant modes for near-field heat enhancement, regardless of magnetic response. The total heat flux is further enhanced with dual uniaxial electromagnetic metamaterials. As discussed previously in Figs. 3(c) and 3(d) for $p$ polarization, the uniaxial metamaterial with property set I has broadband type 2 e-HM mode at frequencies $\omega<\omega_{\mathrm{p}}$, while the uniaxial metamaterial with property set II exhibits broadband type $1 \mathrm{e}-\mathrm{HM}$ mode at high frequencies $\omega>\omega_{\mathrm{p}}$ and strong coupled e-SPP modes at low frequencies $\omega<\omega_{\mathrm{p}}$. As a result, the uniaxial metamaterial with property set II could achieve the highest total heat flux by 2.5 times more than that with the isotropic materials. Figure 5(c) shows the overall total heat flux for randomly polarized waves by adding those from $\mathrm{s}$ and p polarizations. Clearly, the contribution from s polarized waves are significant and thus not negligible compared with that from $\mathrm{p}$ polarized waves for all three metamaterials but not the non-magnetic isotropic material $(\varepsilon, \mu=1)$. In fact, the combined total heat flux under randomly polarized waves for the dual uniaxial metamaterials with the property set II is about 4 times higher than that with the property set I, which is slightly greater than the flux for the isotropic magnetic metamaterials.

\section{CONCLUSION}

In summary, near-field radiative heat transfer between two uniaxial electromagnetic metamaterials has been theoretically investigated. Besides magnetic response, both electrical permittivity and magnetic permeability were considered to be uniaxial. By studying uniaxial metamaterials with two different material property sets, the results showed that, besides the e$\mathrm{HM}$ and coupled e-SPP modes for p polarized waves, m-HM and coupled $\mathrm{m}$-SPP modes also exist for s polarized waves responsible for the near-field spectral heat flux enhancements. Analytical dispersion relations were used to confirm both coupled m-SPP and e-SPP modes at different polarizations. Furthermore, the near-field total heat flux for $\mathrm{s}$ polarized waves, which is comparable to that for $\mathrm{p}$ polarized waves and thus cannot be neglected for uniaxial electromagnetic metamaterials, can reach at most 6 times higher than that for isotropic magnetic materials at vacuum gaps less than $1 \mu \mathrm{m}$. The insights gained here would lead to the finding of a new class of dual uniaxial metamaterials in the near-field radiative transfer for various applications.

\section{ACKNOWLEDGMENTS}

The authors are grateful to the support from the National Science Foundation under CBET-1454698 and the New Faculty Startup Program at ASU.

\section{APPENDIX A: THE DERIVATION OF FRESNEL REFLECTION AND TRANSMISSION COEFFICIENTS BETWEEN TWO ARBITRARY DUAL UNIAXIAL ELECTROMAGNETIC MEDIA}

\section{For s polarized waves}

The permittivity and permeability tensors of an arbitrary dual uniaxial electromagnetic medium $i$ can be defined as

$$
\hat{\varepsilon}_{i}=\left[\begin{array}{ccc}
\varepsilon_{i \|} & 0 & 0 \\
0 & \varepsilon_{i \|} & 0 \\
0 & 0 & \varepsilon_{i \perp}
\end{array}\right] \text { and } \hat{\mu}_{i}=\left[\begin{array}{ccc}
\mu_{i \|} & 0 & 0 \\
0 & \mu_{i \|} & 0 \\
0 & 0 & \mu_{i \perp}
\end{array}\right] .
$$

Here, $\|$ and $\perp$ denote the direction parallel (in $x-y$ plane) and perpendicular ( $\mathrm{z}$ direction) to the interface, respectively. The nonzero components of the electric and magnetic fields for $\mathrm{s}$ polarized waves are ${ }^{23}$

$$
E_{y}= \begin{cases}\left(E_{I} e^{i \gamma_{i}^{s} z}+E_{R} e^{-i \gamma_{i}^{s} z}\right) e^{i \beta x} & \text { for } z<0 \\ E_{T} e^{-i \gamma_{j}^{s} z} e^{i \beta x} & \text { for } z>0,\end{cases}
$$

and

$$
H_{x}= \begin{cases}-\frac{\gamma_{i}^{s}}{\omega \mu_{0} \mu_{i \|}}\left(E_{I} e^{i \gamma_{i}^{s} z}-E_{R} e^{-i \gamma_{i}^{s} z}\right) e^{i \beta x} & \text { for } z<0 \\ \frac{\gamma_{j}^{s}}{\omega \mu_{0} \mu_{j \|}} E_{T} e^{i \gamma_{j}^{s} z} e^{i \beta x} & \text { for } z>0 .\end{cases}
$$

Since both $E_{y}$ and $H_{x}$ should be continuous at the boundary $z=0$, Eqs. (A2) and (A3) yield

$$
\left(E_{I}+E_{R}\right) e^{i \beta x}=E_{T} e^{i \beta x},
$$

and

$$
-\frac{\gamma_{i}^{s}}{\omega \mu_{0} \mu_{i \|}}\left(E_{I}-E_{R}\right) e^{i \beta x}=\frac{\gamma_{j}^{s}}{\omega \mu_{0} \mu_{j \|}} E_{T} e^{i \beta x} .
$$

Therefore, with the definition of Fresnel reflection and transmission coefficients for s polarized waves where $r_{i j}^{s}=E_{R} / E_{I}$ and $t_{i j}^{s}=E_{T} / E_{I}$, the expression of the two coefficients can then be derived from Eqs. (A4) and (A5), 


$$
r_{i j}^{s}=\frac{\mu_{j \|} \gamma_{i}^{s}-\mu_{i \|} \gamma_{j}^{s}}{\mu_{j \|} \gamma_{i}^{s}+\mu_{i \|} \gamma_{j}^{s}}
$$

and

$$
t_{i j}^{s}=\frac{2 \mu_{j \|} \gamma_{i}^{s}}{\mu_{j \|} \gamma_{i}^{s}+\mu_{i} \gamma_{j}^{s}}
$$

\section{For p polarized waves}

Similar to the process presented in Appendix A.1, the nonzero components of the electric and magnetic fields for $p$ polarized waves are ${ }^{23}$

$$
E_{x}= \begin{cases}-\frac{\gamma_{i}^{p}}{\omega \varepsilon_{0} \varepsilon_{i} \|}\left(H_{I} e^{i \gamma_{i}^{p} z}-H_{R} e^{-i \gamma_{i}^{p} z}\right) e^{i \beta x} & \text { for } z<0 \\ \frac{\gamma_{j}^{p}}{\omega \varepsilon_{0} \varepsilon_{j} \|} H_{T} e^{i \gamma_{j}^{p} z} e^{i \beta x} & \text { for } z>0,\end{cases}
$$

and

$$
H_{y}= \begin{cases}\left(H_{I} e^{i \gamma_{i}^{p} z}+H_{R} e^{-i \gamma_{i}^{p} z}\right) e^{i \beta x} & \text { for } z<0 \\ H_{T} e^{-i \gamma_{j}^{p} z} e^{i \beta x} & \text { for } z>0 .\end{cases}
$$

Since both $E_{x}$ and $H_{y}$ should be continuous at the boundary $z=0$, Eqs. (A8) and (A9) yield

$$
-\frac{\gamma_{i}^{p}}{\omega \varepsilon_{0} \varepsilon_{i \|}}\left(H_{I}-H_{R}\right) e^{i \beta x}=\frac{\gamma_{j}^{p}}{\omega \varepsilon_{0} \varepsilon_{j \|}} H_{T} e^{i \beta x},
$$

and

$$
\left(H_{I}+H_{R}\right) e^{i \beta x}=H_{T} e^{i \beta x} .
$$

Therefore, with the definition of Fresnel reflection and transmission coefficients for $\mathrm{p}$ polarized waves where $r_{i j}^{p}=$ $H_{R} / H_{I}$ and $t_{i j}^{p}=H_{T} / H_{I}$, the expression of the two coefficients can then be derived from Eqs. (A10) and (A11),

$$
r_{i j}^{p}=\frac{\varepsilon_{j \|} \gamma_{i}^{p}-\varepsilon_{i \|} \gamma_{j}^{p}}{\varepsilon_{j} \| \gamma_{i}^{p}+\varepsilon_{i \|} \gamma_{j}^{p}},
$$

and

$$
t_{i j}^{p}=\frac{2 \varepsilon_{j \|} \gamma_{i}^{p}}{\varepsilon_{j \|} \gamma_{i}^{p}+\varepsilon_{i} \gamma_{j}^{p}} .
$$

\section{The Fresnel reflection and transmission coefficients at the interface between vacuum and a dual uniaxial electromagnetic medium}

Let us assume medium 0 is vacuum and medium 2 is a dual uniaxial electromagnetic medium, Eqs. (A6) and (A7) can be simplified by $\mu_{0 \|}=\mu_{0 \perp}=\mu_{0}=1$ and $\gamma_{0}^{s}=\gamma_{0}$ for $\mathrm{s}$ polarized waves

$$
r_{02}^{s}=\frac{\mu_{2 \|} \gamma_{0}-\mu_{0} \gamma_{2}^{s}}{\mu_{2 \|} \gamma_{0}+\mu_{0} \gamma_{2}^{s}}=\frac{\mu_{2 \|} \gamma_{0}-\gamma_{2}^{s}}{\mu_{2 \|} \gamma_{0}+\gamma_{2}^{s}},
$$

and

$$
t_{02}^{s}=\frac{2 \mu_{2 \|} \gamma_{0}}{\mu_{2 \|} \gamma_{0}^{s}+\mu_{0} \gamma_{2}^{s}}=\frac{2 \mu_{2 \|} \gamma_{0}}{\mu_{2 \|} \gamma_{0}^{s}+\gamma_{2}^{s}} .
$$

For $\mathrm{p}$ polarized waves where $\varepsilon_{0 \|}=\varepsilon_{0 \perp}=\varepsilon_{0}=1$ and $\gamma_{0}^{p}=\gamma_{0}$, Eqs. (A12) and (A13) yield

$$
r_{02}^{p}=\frac{\varepsilon_{2 \|} \gamma_{0}-\varepsilon_{0} \gamma_{2}^{p}}{\varepsilon_{2 \|} \gamma_{0}+\varepsilon_{0} \gamma_{2}^{p}}=\frac{\varepsilon_{2 \|} \gamma_{0}-\gamma_{2}^{p}}{\varepsilon_{2 \|} \gamma_{0}+\gamma_{2}^{p}},
$$

and

$$
t_{02}^{p}=\frac{2 \varepsilon_{2 \|} \gamma_{0}}{\varepsilon_{2 \|} \gamma_{0}+\varepsilon_{0} \gamma_{2}^{p}}=\frac{2 \varepsilon_{2 \|} \gamma_{0}}{\varepsilon_{2 \|} \gamma_{0}+\gamma_{2}^{p}} .
$$

\section{APPENDIX B: THE DERIVATION OF COMPONENT OF THE WAVEVECTOR VERTICAL TO THE INTERFACE IN AN ARBITRARY DUAL UNIAXIAL ELECTROMAGNETIC MEDIUM}

The wavevector $k$ can be separated into two components: component parallel to the interface $\beta$ and component vertical to the interface $\gamma$. Therefore, the relationship between wavevector and the two components of medium $i$ can be expressed as $\beta_{i}{ }^{2}+\gamma_{i}^{2}=\left|k_{i}\right|^{2}=n_{i}{ }^{2} \omega^{2} / c_{0}^{2}$. According to Ref. 24,

$$
\frac{\beta_{i}{ }^{2} \varepsilon_{i \|} \mu_{i \|}}{n_{i}{ }^{2}-\varepsilon_{i \|} \mu_{i \|}}+\frac{\gamma_{i}{ }^{2} \varepsilon_{i \perp} \mu_{i \perp}}{n_{i}{ }^{2}-\varepsilon_{i \perp} \mu_{i \perp}}=0 .
$$

By combining the two equations, Eq. (B1) yields

$$
\begin{aligned}
n_{i}{ }^{2} \beta_{i}{ }^{2} \varepsilon_{i \|} \mu_{i \|}+n_{i}{ }^{2} \gamma_{i}{ }^{2} \varepsilon_{i \perp} \mu_{i \perp} & =\varepsilon_{i \perp} \mu_{i \perp} \varepsilon_{i \|} \mu_{i \|}\left(\beta_{i}{ }^{2}+\gamma_{i}{ }^{2}\right) \\
& =\varepsilon_{i \perp} \mu_{i \perp} \varepsilon_{i \|} \mu_{i \|}\left(\frac{n_{i}{ }^{2} \omega^{2}}{c_{0}^{2}}\right) .
\end{aligned}
$$

Then Eq. (B2) yields the general equation of the wavevector component vertical to the interface

$$
\gamma_{i}=\sqrt{\varepsilon_{i \|} \mu_{i \|}\left(\frac{\omega^{2}}{c_{0}^{2}}\right)-\frac{\varepsilon_{i \|} \mu_{i \|}}{\varepsilon_{i \perp} \mu_{i \perp}} \beta_{i}{ }^{2}} .
$$

\section{For s polarized waves}

For s polarized waves, medium $i$ can be considered as isotropic for electric field. Therefore, the two components of permittivity tensor are the same $\left(\varepsilon_{\|}=\varepsilon_{\perp}\right)$ and Eq. (B3) yields the vertical component of wavevector for s polarized waves

$$
\gamma_{i}^{s}=\sqrt{\varepsilon_{i \|} \mu_{i \|} \frac{\omega^{2}}{c_{0}^{2}}-\frac{\mu_{i \|}}{\mu_{i \perp}} \beta_{i}{ }^{2}} .
$$

\section{For p polarized waves}

Similarly, medium $i$ can be considered as isotropic for magnetic field with $\mathrm{p}$ polarized waves. Therefore, with $\mu_{\|}=\mu_{\perp}$, Eq. (B3) yields the vertical component of wavevector for $\mathrm{p}$ polarized waves 


$$
\gamma_{i}^{p}=\sqrt{\varepsilon_{i \|} \mu_{i \|} \frac{\omega^{2}}{c_{0}^{2}}-\frac{\varepsilon_{i \|}}{\varepsilon_{i \perp}} \beta_{i}{ }^{2}} .
$$

\section{APPENDIX C: THE DERIVATION OF SINGLE INTERFACE SPP CONDITION FOR DUAL UNIAXIAL ELECTROMAGNETIC MEDIA}

For the interface between air and a dual uniaxial electromagnetic metamaterial, the dispersion curve of single interface $\mathrm{m}-\mathrm{SPP}$ can be calculated by

$$
\mu_{1 \|} \gamma_{0}+\gamma_{1}^{s}=0
$$

where

$$
\gamma_{0}^{s}=\sqrt{\omega^{2} / c^{2}-\beta^{2}}
$$

and

$$
\gamma_{1}^{s}=\sqrt{\varepsilon_{1 \|} \mu_{1 \|} \omega^{2} / c^{2}-\frac{\mu_{1 \|}}{\mu_{1 \perp}} \beta^{2}} .
$$

Therefore, Eq. (C1) yields

$$
\mu_{1 \|} \sqrt{\omega^{2} / c^{2}-\beta^{2}}+\sqrt{\varepsilon_{1 \|} \mu_{1 \|} \omega^{2} / c^{2}-\frac{\mu_{1 \|}}{\mu_{1 \perp}} \beta^{2}}=0 .
$$

By assuming $\beta \gg \omega / c$, Eq. (C4) yields $\mu_{\|} \mu_{\perp}=1$. Similarly, the dispersion curve of single interface e-SPP between air and a dual uniaxial electromagnetic metamaterial can be obtained by

$$
\varepsilon_{1 \|} \gamma_{0}+\gamma_{1}^{p}=0
$$

By plugging in the equations of $\gamma_{0}$ and $\gamma_{1}{ }^{p}$ along with the assumption of $\beta \gg \omega / \mathrm{c}$, the condition $\varepsilon_{\|} \varepsilon_{\perp}=1$ can be derived for e-SPP.

${ }^{1}$ K. Park, S. Basu, W. P. King, and Z. M. Zheng, J. Quant. Spectrosc. Radiat. Transfer 109, 305 (2008).

${ }^{2}$ J.-Y. Chang, Y. Yang, and L. P. Wang, Int. J. Heat Mass Transfer 87, 237 (2015).

${ }^{3}$ Y. Yang, S. Basu, and L. P. Wang, Appl. Phys. Lett. 103, 163101 (2013).

${ }^{4}$ Y. Yang, S. Basu, and L. P. Wang, J. Quant. Spectrosc. Radiat. Transfer 158, 69 (2015).

${ }^{5}$ S. Basu and L. P. Wang, Appl. Phys. Lett. 102, 053101 (2013).

${ }^{6}$ X. L. Liu, L. P. Wang, and Z. M. Zhang, Nanoscale Microscale Thermophys. Eng. 19, 98 (2015).

${ }^{7}$ K. Park and Z. M. Zhang, Front. Heat Mass Transfer 4, 013001 (2013).

${ }^{8}$ S.-A. Biehs, M. Tschikin, and P. Ben-Abdallah, Phys. Rev. Lett. 109, 104301 (2012).

${ }^{9}$ X. L. Liu, R. Z. Zhang, and Z. M. Zhang, Appl. Phys. Lett. 103, 213102 (2013).

${ }^{10}$ Y. Guo, C. L. Cortes, S. Molesky, and Z. Jacob, Appl. Phys. Lett. 101, 131106 (2012).

${ }^{11}$ Y. Guo, W. Newman, C. L. Cortes, and Z. Jacob, Adv. Optoelectron. 2012, 452502.

${ }^{12}$ Z. Jacob, MRS Bull. 37, 761 (2012).

${ }^{13}$ C. L. Cortes, W. Newman, S. Molesky, and Z. Jacob, J. Opt. 14, 063001 (2012).

${ }^{14}$ L. P. Wang and Z. M. Zhang, Appl. Phys. Lett. 95, 111904 (2009).

${ }^{15}$ L. P. Wang and Z. M. Zhang, J. Opt. Soc. Am. B 27, 2595 (2010).

${ }^{16}$ L. P. Wang and Z. M. Zhang, Appl. Phys. Lett. 100, 063902 (2012).

${ }^{17}$ L. P. Wang and Z. M. Zhang, J. Heat Transfer 135, 091505 (2013).

${ }^{18}$ S. Basu and M. Francoeur, Opt. Lett. 39, 1266 (2014).

${ }^{19}$ J. B. Pendry, A. J. Holden, D. J. Robbins, and W. J. Stewart, IEEE Trans. Microwave Theory Tech. 47, 2075 (1999).

${ }^{20}$ X. L. Liu, T. J. Bright, and Z. M. Zhang, J. Heat Transfer 136, 092703 (2014).

${ }^{21}$ S. Basu, Z. M. Zhang, and C. J. Fu, Int. J. Energy Res. 33, 1203 (2009).

${ }^{22}$ X. L. Liu, R. Z. Zhang, and Z. M. Zhang, Int. J. Heat Mass Transfer 73, 389 (2014).

${ }^{23}$ Z. M. Zhang, Nano/microscale Heat Transfer (McGraw-Hill, New York, 2007).

${ }^{24}$ C. C. Davis, Lasers and Electro-optics: Fundamentals and Engineering (Cambridge University Press, Cambridge, England, 1996), p. 591. 